\title{
MICROFITÓLITOS ASSOCIADOS A CONSTRUÇÕES ESTROMATOLÍTICAS DO GRUPO BAMBUÍ, PROTEROZÓICO SUPERIOR, NA REGIÃO DE ARCOS - MG.
}

\author{
JANE NOBRE \& ARMANDO MÁRCIO COIMBRA (IN MEMORIAM)
}

\begin{abstract}
MICROPHYTOLITES ASSOCIATED WITH STROMATOLITIC BUILD'UPS OF THE BAMBUI GROUP, LATE PROTEROZOIC, IN THE ARCOS REGION - MG. The Neoproterozoic Bambuif Group consists in the Arcos region of a sucession of $120 \mathrm{~m}$ of carbonate rocks withouth associated siliciclastic rocks. Four main shallowing upward intervals were identified in the area: (1) a basal interval represented by a carbonate ramp with beach-barrier-lagoon in the inner ramp and evidence of subaerial exposure in the upper part of the interval; (2) stromatolitic interval consisting of several, few meters thick, shallowing upward cycles, with common intercalations of detritic carbonate horizons with cross-bedded microphytolitic grainstones-to-packstones up to $1.5 \mathrm{~m}$ thick and few horizons of rudstones composed of intraformational dolomite flakes derived from the stromatolites. The stromatolites are mostly stratiform to pseudocolumnar; biostromes are dominant, with rare bioherms of columnar or dome-shaped stromatolites. The entire interval is dolomitized and the dolomite texturally preserves crypto-to-fine crystalline; anhydrite occurs scattered with no particular concentration zones. Intervals 3 and 4 are very similar to each other and resemble Oolite-Grainstone Cycles; the upper facies of Interval 3 is similar to Lime-mud-Sabkha Cycles.

Microphytolites, as used in the paleontological literature, refer to a great variety of carbonatic grains such as oncoids, microbial lumps and catagraphs. They are restricted in time and were most important in Proterozoic times. They constitute an especial kind of grain with a complex, polygenetic origin, resulting from interaction between microbial activity and sedimentary/diagenetic processes, and are considered as paleoenvironmental indicators reflecting intermediate, moderate, energy conditions. In the Arcos region, microphytolites are found in Interval 2 , and consist of catagraphs, most of them similar to Vesicularites and Vermiculites. They are usually associated with columnar stromatolites or constitute cross-bedded dolarenite. Wherever related to stromatolites, they compose part of the laminations and the walls, and exibhit some morphological differences as compared to the detrital ones. They also may fill the intercolumnar space between stromatolites. The microphytolites of Mina da Bocaina afford new insights into Proterozoic paleoenvironments and reflect moderate energy conditions.

Keywords: Microphytolite, stromatolite, Proterozoic paleoenvironments, carbonates, Bambuí Group.
\end{abstract}

RESUMO O Grupo Bambuí (Neoproterozóico) consiste de duas seqüências principais: uma inferior, marinha, carbonática/terrígena e uma superior, terrígena, continental Na região de Arcos (sudoeste mineiro) ocorrem apenas carbonatos representativos da seqüência inferior (Subgrupo Paraopeba) que configuram um megaciclo regressivo composto por quatro intervalos principais de sedimentação. O Intervalo $I$ representa uma rampa carbonática com barreira arenosa. O Intervalo 2, composto por ciclos regressivos métricos, é eminentemente estromatolítico e apresenta intercalações de dolarenitos com microfitólitos; sedimentos terrígenos estão ausentes. É interpretado como tendo se desenvolvido em planície de maré, numa plataforma carbonática de baixa declividade. Os microfitólitos apresentam grande diversidade morfológica e ocorrem: 1) associados a estromatólitos, colunares em especial, onde constituem parte das laminações, a parede lateral e/ou preenchem os espaços intercolunares; 2) como principal componente dos dolarenitos (grainstones) que ocorrem intercalados nas construções estromatolíticas. Os intervalos 3 e 4 mostram semelhanças com ciclos Oolite-Grainstones.

O termo microfitólito, como utilizado na literatura paleontológica do Proterozóico, designa uma grande variedade de corpúsculos carbonáticos, entre eles oncóides, microbial lumps e catagraphs. Apresentam restrição temporal, com acentuado declínio no Fanerozóico e foram muito utilizados em bioestratigrafia. Como sua origem atualmente é considerada como decorrente da interação entre microorganismos, processos sedimentares e diagenéticos, a utilização de microfitólitos para essa finalidade fica comprometida. Podem contudo ser utilizados como marcadores paleoambientais, sugerindo condições de energia moderada.

Palavras-chaves: microfitólitos, estromatólitos, indicadores paleoambientais, Proterozóico, carbonatos, Grupo Bambuí.

INTRODUÇÃo O registro fóssil do Proterozóico, embora não apresente a diversidade paleontológica encontrada nas unidades faneroź́icas, é bastante rico e microfitólitos fazem parte desse acervo como "fósseis" clássicos desse período da história geológica.

O Grupo Bambuí, remanescente de uma ampla bacia sedimentar neoproterozóica, tem sua principal área de ocorrência ao longo do rio São Francisco. O registro fóssil aí conhecido é composto por bioconstruções, estromatolíticas ou não, microfósseis planctônicos e microfitólitos.

Este trabalho versa sobre microfitólitos que ocorrem na Mina da Bocaina - CSN próximo à cidade de Arcos, no sudoeste do estado de Minas Gerais. Serão abordados segundo o seu aspecto morfológico, o ambiente sedimentar onde se desenvolveram e seu significado paleoambiental.

Os principais padrões de referência utilizados para a descrição e comparação dos microfitólitos foram os trabalhos de Sweet \& Knoll (1985) no Draken Conglomerate (Spitsbergen, Dinamarca), BertrandSarfati (1972) no Sahara norte-ocidental e Zhuravleva (1963) na antiga União Soviética.

ESTRATIGRAFIA O Grupo Bambuí (Rimann 1917) insere-se no Supergrupo São Francisco (Pflug \& Renger 1973) e constitui uma extensa cobertura de plataforma depositada sobre o Cráton do São Francisco. É composto por duas seqüências principais: a inferior, marinha, carbonática/terrígena, representada pelo Subgrupo Paraopeba (formações Sete Lagoas, Serra de Santa Helena, Lagoa do Jacaré e Serra da Saudade) e a superior, terrígena, de natureza continental, denominada Formação Três Marias (Projeto RADAMBRASIL 1982).

A divisão atual do grupo, com algumas modificações, segue a proposição inicial de Costa \& Branco (1961) e Dardenne (1978). A principal controvérsia é a exclusão (Couto \& Bez 1981, RADAMBRASIL 1982) dos sedimentos diamictíticos da Formação Jequitaí e unidades cronocorrelatas, ou sua manutenção (Dardenne 1978), na base do Grupo Bambuí.
Na região de Arcos ocorre apenas a seqüência inferior (Subgrupo Paraopeba), que é exclusivamente carbonática; representa um megaciclo regressivo (Nobre-Lopes 1995), composto por quatro intervalos principais de sedimentação (Fig. 2).

O Intervalo 1, basal, representa uma plataforma carbonática progradante do tipo rampa com barreira arenosa; fácies distais (rampa externa) são compostas por construções microbianas não estromatolíticas (biostromas) e depósitos de tempestades. Nas fácies proximais (rampa interna e back ramp) predominam carbonatos detríticos (dolomitizados) os quais compõem shoals, com tempestitos associados e bioconstruções estromatolíticas. Um evento de exposição subaérea afetou as fácies de topo do Intervalo 1 gerando dissolução, com abertura de cavidades e formação de brechas de dissolução/colapso.

O Intervalo 2 é eminentemente estromatolítico e representa uma planície de maré regressiva que se instalou em plataforma carbonática de baixa declividade, após o evento de exposição subaérea que afetou o Intervalo 1. Dolomitos estromatolíticos com intercalações regulares de dolarenitos/ dolorruditos com microfitólitos e fragmentos de estromatólitos predominam em todo o intervalo e compõem sucessões menores de caráter regressivo.

Os intervalos 3 e 4 , de topo, são semelhantes a ciclos Oolite Grainstone, característicos de plataformas rasas e amplas, com corpos de calcarenitos oolíticos nas suas porções superiores. As fácies de topo do Intervalo 3 mostram ainda semelhanças com ciclos do tipo Line Mud-Sabkha..

Microfósseis planctônicos, ACRITARCHA, identificados em calcissiltitos argilosos próximo à base do pacote carbonático sugerem idade Rifeano Superior/Vendiano (Cruz \& Nobre-Lopes 1992); uma única forma encontrada e não identificada taxonomicamente apresenta ornamentos (espinhos), o que poderia sugerir idade mais próxima do limite Precambriano-Cambriano. 


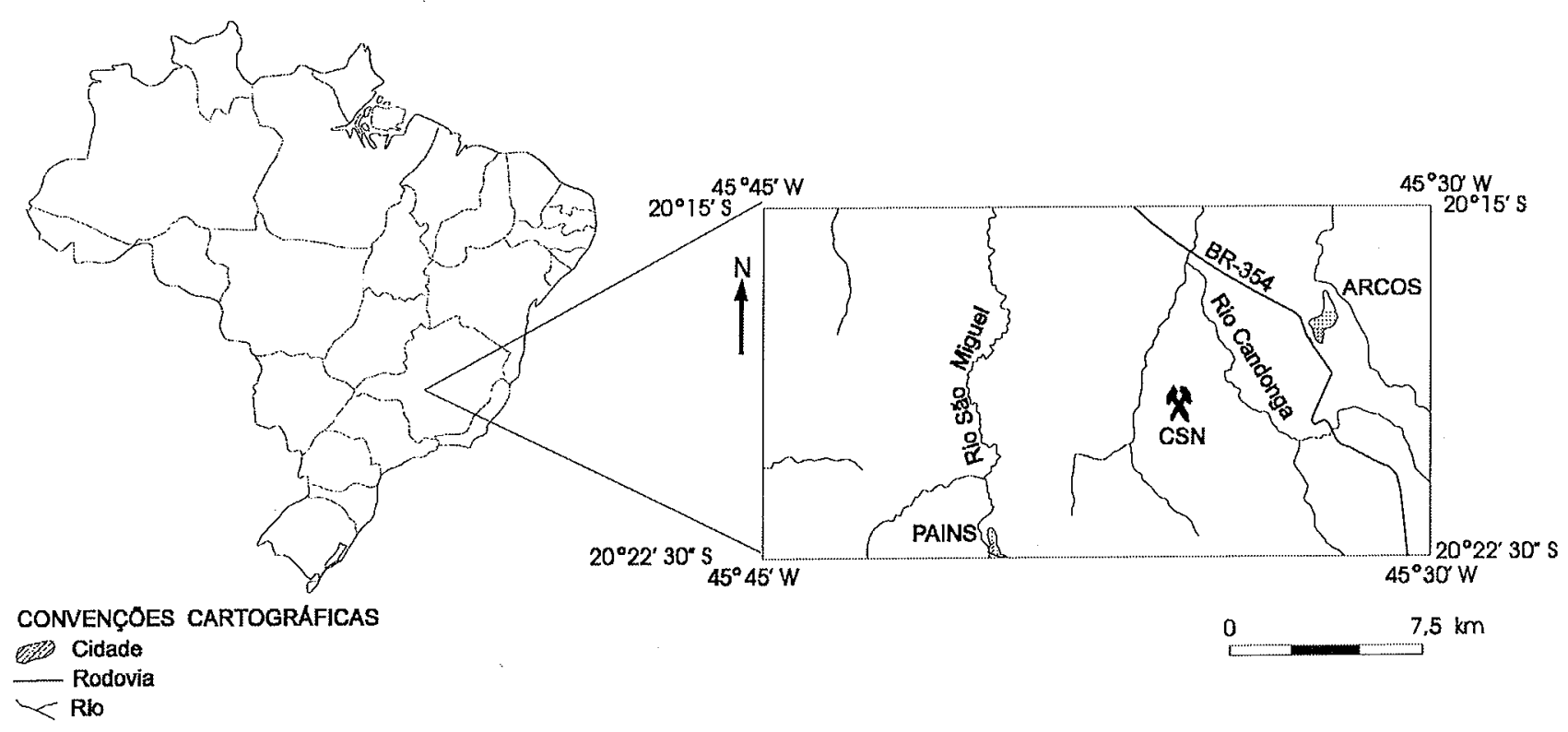

Figura I-Mapa de localizą̧ão da área.

INTERVALO 2 Petrograficamente o Intervalo 2 é composto por dolomitos cinza claros, de cristalinidade fina, que preservam em grande parte a textura original dos níveis bioconstruídos e sedimentos associados.

A seção-tipo descrita é a da Mina da Bocaina (Fig. 2) por apresentar melhor área de observação e exposição contínua (Nobre-Lopes 1995). Bioconstruções estromatolíticas, biostromas no geral, perfazem cerca de $90 \%$ de todo o intervalo que tem cerca de $25 \mathrm{~m}$ de espessura. Compõem ciclos regressivos métricos que apresentam no topo gretas de ressecação, níveis microbianos rompidos e estruturas geopetais relacionadas a diagênese vadosa; truncamentos por grandes marcas onduladas também são comuns. Cristais de evaporitos, em especial anidrita, são regularmente observados, embora em pequena quantidade.

Os níveis granulares e/ou placóides que ocorrem como intercalações nas bioconstruções têm espessura máxima observada de $1.5 \mathrm{~m}$; os contatos com as bioconstruções são nítidos e regulares. Estratificação cruzada por ondas e marcas onduladas (decimétricas a centimétricas) são as estruturas sedimentares mais freqüentes.

Nos biostromas predominam estromatólitos estratiformes a pseudocolunares com laminação plana a ondulada; eventualmente observa-se ainda formas dômicas e colunares. Biohermas ocorrem subordinadamente e são constituídos por estromatólitos pseudocolunares a colunares, com alguns elementos dômicos associados.

As intercalações de dolarenitos a microfitólitos compõem grainstones, mais raramente packstones e têm arcabouço aberto, sem evidências de compactação. Entre os aloquímicos predominam microfitólitos, mas observa-se ainda pelóides, oólitos superficiais micríticos e agregados botrioidais; a granulação varia de areia muito fina a grânulos e os grãos apresentam vestígios de franja isópaca acicular. Dolorruditos no geral são constituídos por fragmentos lamelares de estromatólitos, e se encontram intercalados, como níveis decimétricos, em estromatólitos estratiformes. Texturalmente variam de rudstones a floatstones.

A seguir serão descritos os $3 \mathrm{~m}$ basais aflorantes do Intervalo 2, os quais constituem um ciclo regressivo menor.

$\mathrm{Na}$ base predominam maciços estromatolíticos de aspecto arborescente que gradam a biostroma composto por estromatólitos estratiformes a pseudocolunares com elementos colunares associados. Essas bioconstruções são recobertas por um nível de dolarenito a microfitólitos ao qual se sobrepõem estromatólitos estratiformes

Os maciços estromatolíticos basais têm cerca de 80 centímetros de altura e são constituídos por elementos colunares a pseudocolunares; inicialmente predominam formas dômicas ou colunares, com relevo positivo e parede lateral que desaparecem à medida que os estromatólitos se desenvolvem. As laminações são sempre espessas, apresentam baixo grau de herança e quantidades variáveis de materi-
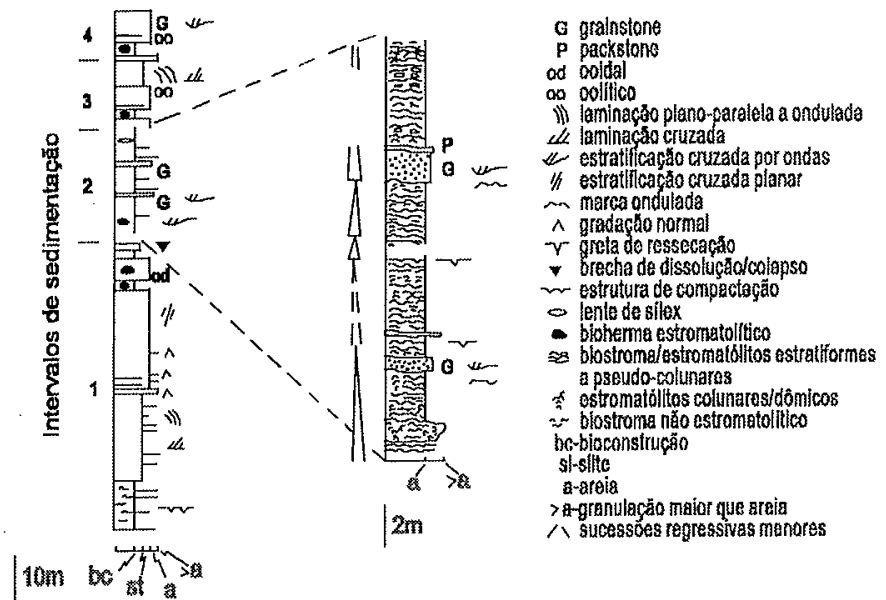

Figura 2 - Perfil simplificado da Mina da Bocaina (CSN) - Arcos $M G$.

al granular, pelóides em especial. Lateralmente aos maciços estromatolíticos as estruturas são menos proeminentes e a quantidade de pelóides é muito maior, formando inúmeros níveis de grainstones. Não se observa lama carbonática associada a esse nível.

As formas colunares que ocorrem no biostroma que capeia os maciços arborescentes, são decimétricas, ligeiramente oblíquas, assimétricas e possuem parede lateral. Microfitólitos constituem não só parte das laminaçóes, mas também a parede lateral dos estromatólitos, onde se aglutinam verticalmente; preenchem ainda os espaços intercolunares constituindo grainstones. É comum a presença de cristais aciculares de anidrita.

$\mathrm{O}$ dolarenito composto por microfitólitos que capeia as bioconstruções tem cerca de $70 \mathrm{~cm}$ de espessura e apresenta estratificação cruzada por ondas compondo sets centimétricos a decimétricos com marcas onduladas decimétricas. Observa-se ainda truncamentos por ondulações métricas. Texturalmente constitui um grainstone mal selecionado, com predomínio das frações areia grossa a muito grossa e arcabouço aberto. Os microfitólitos apresentam vestígios de franja isópaca acicular.

Laminação fenestral e gretas de ressecação são feições comumente observadas nos estromatólitos estratiformes que compõem a unidade de topo.

Interpretação O Intervalo 2 representa a instalação de uma plataforma carbonática progradante, de águas rasas, com planícies de marés em direção à linha de costa após o evento de exposição subaérea (Nobre-Lopes 1995). É composto por sucessões regressivas menores, indicativas de pequenas oscilações do nível do mar. O ciclo basal des- 
crito representa uma sucessão regressiva menor, onde as construções estromatolíticas arborescentes representam a fácies mais distal, gradando, em direção à linha de costa, aos estromatólitos com microfitólitos associados, ao dolarenito a microfitólitos e aos estromatólitos estratiformes com gretas de ressecação. Os estromatólitos em maciços arborescentes representariam uma construção recifal desenvolvida em ambiente de inframaré, com águas claras e agitadas: o aporte irregular de carbonato detrítico, arenoso, sugere a ação de eventos episódicos, como tempestades ou marés de maior energia; a ausência de material lamoso nas laminações estromatolíticas também é sugestiva de ambiente de águas agitadas. A barreira constituída pelos maciços estromatolíticos teria gerado um ambiente mais protegido em direcão à linha de costa, com águas menos agitadas, mas ainda sujeitas à ação de marés e eventos de tempestade (estratificações cruzadas por ondas, marcas onduladas decimétricas e truncamentos por ondulações maiores). A presença de anidrita sugere condições eventuais de alta evaporação. Os estromatólitos que apresentam estreita associação com microfitólitos teriam se desenvolvido próximo aos estromatólitos em maciços arborescentes, mas em ambiente mais protegido. Em ambiente ainda de inframaré foi depositado o dolarenito a microfitólitos cujos grãos apresentam vestígios de franja isópaca acicular, característica de ambiente marinho freático. A ausência de feições que indiquem diagênese vadosa também reforça essa interpretação. Em ambiente de intermaré (alta?) desenvolveram-se os estromatólitos estratiformes de topo, com laminação fenestral e gretas de ressecação.

MICROFITÓLITOS Microfitólito (microphytolite), termo utilizado na literatura paleontológica do Proterozóico, designa uma grande variedade de grãos carbonáticos tais como oóides, oncóides, microbial lumps, grapestones e outros de difícil catalogação, que foram denominados Catagraphia por Maslov (1953) e incluídos na Família dos Catagraphs. Atualmente o termo Catagraph não é mais utilizado com a conotação de família, mas identifica um grupo de "corpúsculos carbonáticos de dimensões diversas, isolados ou aglutinados, que não apresentam envelopes concêntricos" (Bertrand-Sarfati 1972). São subdivididos em tipos específicos e foram muito utilizados por diversos autores em bioestratigrafia, com denominação binomial, lineana. Tucker \& Wright (1990) utilizam o termo Catagraph com a conotação de grãos algais calcitizados.

Segundo Swett \& Knoll (1985) microfitólitos são grãos geneticamente compostos, derivados do crescimento de bactérias ou cianobactérias em associação com processos de mineralização inorgânica, abrasões periódicas, eventos erosivos e processos neomórficos. Embora sejam característicos do Proterozóico, os autores sugerem, baseados em Monty (1976), que grãos morfologicamente semelhantes podem ser encontrados em ambientes recentes, relacionados à formação de carbonatos microbianos de fabrics trombolíticos Cabe ressaltar que Monty (op. cit.) em nenhum momento faz correlação ou comparação entre esses fabrics e microfitólitos. Por representarem o registro de presumível atividade biológica, os microfitólitos podem ser considerados como bioclastos, embora com conotação algo diferente da utilizada em rochas fanerozóicas.

Microfitólitos foram descritos em carbonatos do Proterozóico Médio/Superior em várias regiões do mundo como na antiga União Soviética (Reitlinger 1959, Krylov 1963, Zhuravleva 1963), Groelândia (Bertrand-Sarfati \& Caby 1976,1988 ) e norte da Africa (BertrandSarfati 1972). Em Spitsbergen (Dinamarca), foram descritos por Wilson (1961) com a denominação de rocha oolitoidal em três unidades, entre elas o Draken Conglomerate, objeto de estudo do Sweet \& Knoll (1985).

$\mathrm{Na}$ Mina da Bocaina predominam microfitólitos semelhantes a Catagraphs, em especial aos denominados Vesicularites Reitlinger (1959 apud Bertrand-Sarfati 1972, Zhuravleva 1963), que são pequenas vesículas sub-esféricas, ovais ou irregulares que podem ainda formar agregados. Corpúsculos semelhantes a Vermiculites Reitlinger (1959 apud Bertrand-Sarfati 1972) ocorrem subordinadamente e formam agregados irregulares, frequentemente alongados, limitados por um envelope micrítico, escuro.

Os microfitólitos estudados ocorrem: i) como parte das laminações estromatolíticas ou constituindo a parede lateral de estromatólitos colunares; ii) como grainstones, preenchendo os espaços intercolunares nas bioconstruções e iii) compondo espessos corpos de dolarenitos com estratificação cruzada por ondas e marcas onduladas.

Em seção delgada, observa-se que os microfitólitos são muito diversificados (Fig. 3). Podem ser simples, individuais, ou constituir estruturas compostas, aglutinadas (Figs. 3A-3D); podem ainda ser compactos (Fig. 3C) ou extremamente alongados (Figs. 3A e 3D), com projeções e embaiamentos. Apresentam laminações irregulares, com truncamentos evidentes, raramente paralelas (Fig. 3D). Alguns microfitólitos parecem ser "multicamerados" (Figs. 3C e 3D), mas como os descritos por Swett \& Knoll (1985), na maioria das vezes as "câmaras" parecem ser de acresção, não sendo representativas de uma estrutura biológica definida. Ás dimensões variam de $0,11 \mathrm{~mm}$ $2,80 \mathrm{~mm}$ na maior dimensão (areia fina a grânulo). Raramente observase deformação por compactação, o que é indicativo de litificação precoce. Como estão dolomitizados de forma mimética, parte de suas características originais estão preservadas, inclusive vestígios de franja isópaca acicular; pequenas áreas silicificadas são eventualmente observadas, em especial no núcleo dos grãos. Evidências de abrasão são raras.

Observa-se ainda que os microfitólitos que compõem parte dos estromatólitos (laminações e paredes) apresentam morfologias diferenciadas, algumas vezes amebóides e de contornos muito suavemente ondulados se comparados aos que compõem corpos de grainstones e packstones (Figs. 3E e 3F).

CONSIDERAÇÕES FINAIS Os microfitólitos que ocorrem na Mina da Bocaina são muito semelhantes aos descritos pelos diversos autores citados e à exemplo do que ocorre nas demais regiões também estão estreitamente relacionados a bioconstruções estromatolíticas. Como morfologicamente são muito similares aos descritos por Swett \& Knoll (1985), é possível considerá-los como tendo se formado de modo análogo, a partir da interação de processos múltiplos, orgânicos e inorgânicos, não correspondendo portanto a um tipo fóssil definido. As diferenças morfológicas observadas nos microfitólitos que formam laminações e paredes dos estromatólitos colunares e os que compöem corpos de grainstones, não foram descritas no Draken Conglomerate.

Para concluir que microfitólitos podem ser utilizados como indicadores de paleoambiente, sugerindo condições de energia moderada Sweet \& Knoll (1985) se basearam numa série de fatores. Entre os principais estão algumas feições intrínsecas dos grãos, como o não paralelismo das lâminas internas e as poucas evidências de abrasão e a posição dos grainstones a microfitólitos na paleogeografia definida pela associação de fácies em Spitsbergen: os grainstones a microfitólitos representam fácies de energia intermediária, com estratificação cruzada, em uma plataforma que não recebia aporte de terrígenos e apresentava decréscimo de energia em direção à linha de costa $\mathrm{Na}$ Mina da Bocaina, o desenvolvimento do Intervalo 2 ocorreu numa plataforma exclusivamente carbonática, onde os ciclos regressivos exibem um decréscimo de energia em direção à linha de costa; os corpos de dolarenitos a microfitólitos situam-se em condições de energia moderada. Assim, para o ciclo basal descrito, tem o seguinte quadro paleogeográfico partindo de um ambiente distal em direção à linha de costa: 1) maciços estromatolíticos arborescentes que se desenvolve ram em águas agitadas e que por constituírem barreiras recifais propiciaram o desenvolvimento de ambientes mais protegidos, de menor energia, em direção à linha de costa; 2) os estromatólitos colunares com microfitólitos associados desenvolveram-se internamente ao recife; 3) os corpos de dolarenitos com microfitólitos se desenvolveram em ambiente de águas agitadas, mas não de alta energia como indica a presença de estratificações cruzadas por ondas associadas a pequenas marcas onduladas; 4) em ambiente intermaré, próximo à linha de costa, desenvolveram-se os estromatólitos estratiformes de topo, com laminação fenestral e gretas de ressecação.

Os dados acima apresentados reforçam a assertiva de Swett \& Knoll (1985) de que microfitólitos, quando compondo corpos arenosos, podem ser utilizados como marcadores paleoambientais e que seu desenvolvimento se dá, via de regra, em condições intermediárias de energia Como bacias carbonáticas proterozóicas não exibem a grande diversidade biológica observada no Fanerozóico, a possibilidade de utilização de microfitólitos como indicadores paleoambientais é de grande importância.

Agradecimentos À Superintendência da CSN na Mina da Bocaina pela autorização e facilidades no trabalho e em especial ao geol. Antonio Salvador. R. da Silva, pela gentileza e acompanhamento. Agradecemos ainda ao Prof. Dr. M. A. Dardenne pela sugestão do artigo, aos colegas Sérgio L. Martini pelas várias revisões do inglês e figuras, à Dra. Norma M. da C. Cruz pela revisão do texto, Erich Breitag pelo tratamento gráfico das figuras e aos dois revisores anônimos da RBG. 


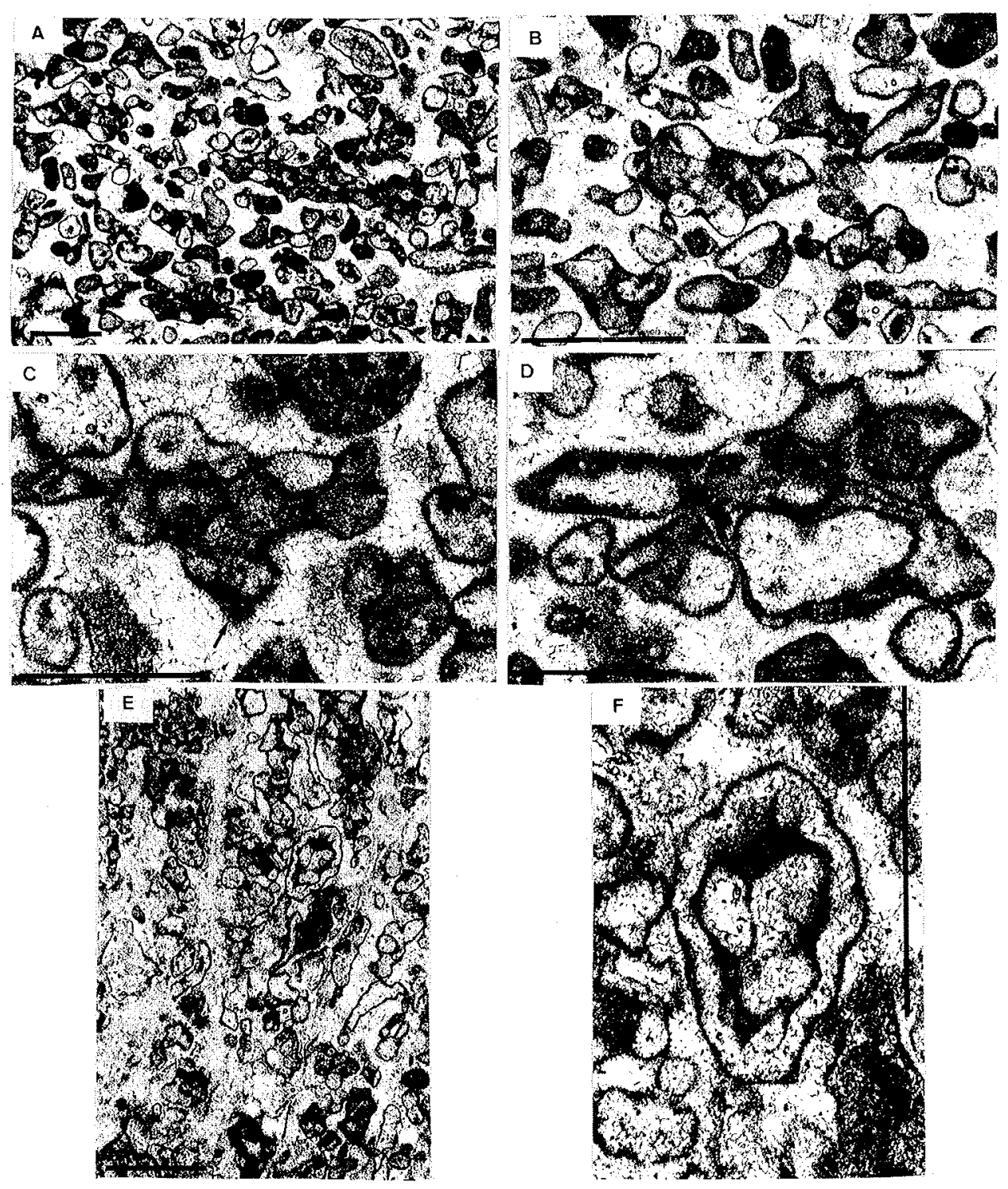

Figura 3 - Fotomicrografias mostrando a variedade morfológica dos microfitólitos, dolomitizados de forma mimética, com preservação da textura dos aloquímicos e neomorfizados. A) Dolarenito (grainstone) composto por microfítólitos, onde se observa a variedade morfológica dos gräas; $B$ ) Microfitólitos simples e compostos, limitados por filme escuro. Presença de franja isópaca acicular: Dolarenito; C) Microfitólito de aspecto macico, "multicamerado", complexo. Presença de franja isópaca acicular e vestígio de airea fibrorradiada (seta). Dolarenito; D) Microfitólito complexo, "multicamerado", no dolarenito. Observar projecóes, embainhamentos e truncamento das láminas escuras; E) Parede lateral de estromatólito colunar composto por microfitólitos. Observar as formas amebóide es contornos suavemente ondulados, incomuns nos microfitólitos que compöem os dolarenitos; F) Detalhe da fotomicrografia E, onde microfitólito "multicamerado" apresenta contomos suavemente ondulados, com laminaçöes escuras nítidas. Todas as fotomicrografias foram obtidas com luz natural, não polarizada. Escala: barra $=1 \mathrm{~mm}$

\section{Referências}

Bertrand-Sarfati J. 1972. Stromatolites columnaires du Precambrien Superieur, Sahara nordoccidental. Inventaire, morphologie et microestructure des laminations, correlation stratigrapiques. Centre de récherche sur les zones" arides (Série Géologie), n.14,

Bertrand-Sarfaty J. \& Caby R. 1976. Carbonates et stromatolites du sommet du Groupe d' Eleonore Bay (Précambrien terminal) au Canning Land (Groenland oriental). Groenlands Geol Underogelse 119.

Bertrand-Sarfaty J. \& Caby R. 1988. The Eleanore Bay Group (central East Greenland). In: Winchester JA. Late proterozoic Stratigraphy of the Northern Atlantic regions. New York Chapman \& Hall p. 212-236.

Costa MT \& Branco JJR. 1961.Introducão.ln: Branco JJR (Ed.) Roteiro para a excursão Belo Horizonte - Brasília. In: SBG, Congresso Brasileiro de Geologia, Belo Horizonte, v. 15, p. $1-119$.

Couto JGP \& Bez L. 1981. A Glaciaç̃o Jequitaí. Um guia estratigráfico para o Precambriano Superior Revista Brasileira de Geociências, 11:17-21.

Cruz NMC \& Nobre-Lopes J. 1992. Microfósseis do Grupo Bambuí na região de Arcos, Minas Gerais. Anais da Academia Brasileira de Ciencias. Rio de Janeiro, v.64, 11.4 p.420. (Resumos)

Dardenne MA. 1978. Síntese sobre a estratigrafia do Grupo Bambuí no Brasil central. In:

Krylov IN. 1963. Stromatolites columnaires ramifiés du Riphéen de Lóural Sud et leur importance pour la stratigraphie du Précambrien supérieur. Trudy Geol. Inst.

S.S.S.R., 69, 133p.
Maslov VP. 1953. Principes de nomenclature et de systématique des stromatolites. lzv. Akad. Nauk., Era. Geol., 4, p. 105-112

Monty CL. 1976. The origin and evolution of cryptalgal fabrics. In: WALTER, M. R. (ed.) sevier, p. $193-249$.

Nobre-Lopes J. 1995. Faciologia e gênese dos carbonatos do grupo Bambui na região de
Arcos, Estado de Minas Gerais. São Paulo, 166p. (Dissertação de Mestrado-InstiArcos, Estado de Minas Gerais. São Paulo,

Pflug R \& Renger FE 1973 Estratigrafia e evoluc̃a geológica da margem SE do Cráton Sanfranciscano. In: SBG, Congresso Brasileiro de Geologia, 27., Aracaju, Ancis, v.2, p. $5-19$.

Radambrasil. 1982. Folha SD.23, Brasília. Levantamento de Recursos Naturais. Rio de Janeiro. v. 29,660 p.

Reitlinger EA. 1959. Atlas of the microscopic organic remains and of the Problemática of the ancient strata in Siberia. Trudy geol. Inst. Akad. Nauk. SSSR, 25, 59p (original em russo).

Rimann E. 1917. A kimberlita no Brasil. Anais da Escola de Minas. Ouro Preto, v. 15, p. $27-32$

Swett K \& Knoll AH. 1985. Stromatolitic bioherms and microphytolites from Late Proterozoic Diaken Conglomeratic Formation. Precambrian Research, 28:327-347. Tucker M \& Wright VP 1990 Carbonate Sedimentology l ed Oxford Blackwell 482p. Wilson CB. 1961. The Upper Middle Hecla Hoek Rocks of Ny Friesland, Spitsbergen Geological Masazine, 48:89-116.

Zhuravleva ZA 1963. Oncolites and Catagraphs of Riphean and Lower Cambrian deposits in some regions of the Siberian Platform. In: Menner VV (Ed) Paleontological basis in some regions of the Siberian Platorm. In: Menner V of the stratigraphy of te Upper Cambrian - Stratigraphy of the USSR, v. 2. Tradudokembriya - pelo Bureau for Translations, Serviço Geológico do Canadá.

Manuscrito A-1067

Recebido em 18 de dezembro de 1998 Revisão dos autores em 31 de janeiro de 2000 Revisano aceita em 5 de fevereiro de 2000 